\title{
ZnO Nanoparticles in Bettering The Color UNIFORMity of Phosphor-ConverTed White LED LIGHTS
}

\author{
Ming-Jui CHEN $N^{1}$, Van Tho LE ${ }^{2}$, Doan Quoc Anh NGUYEN ${ }^{3, *}$, \\ Thinh Cong TRAN $N^{3}$
}

\author{
${ }^{1}$ Department of Electrical Engineering, National Kaohsiung University of Science and \\ Technology, Kaohsiung, Taiwan \\ ${ }^{2}$ Institute of Tropical Biology, Vietnam Academy of Science and Technology, Ho Chi Minh City, \\ Vietnam \\ ${ }^{3}$ Faculty of Electrical and Electronics Engineering, Ton Duc Thang University, Ho Chi Minh \\ City, Vietnam
}

*Corresponding Author: Doan Quoc Anh NGUYEN (Email: nguyendoanquocanh@tdtu.edu.vn)

(Received: 14-Feb-2020; accepted: 20-Jun-2020; published: 30-Sep-2020)

DOI: http://dx.doi.org/10.25073/jaec.202043.280

\begin{abstract}
To make further improvements in future WLED generation, bettering color uniformity is an important goal manufacturers desire to accomplish. One of the most common and effective methods to enhance the color homogeneity is the one focusing on improving scattering in phosphor layer which can be achieved by adding $\mathrm{ZnO}$ into the phosphor layer. Based on theoretical application of Mie-scattering, we compute and analyze the scattering characteristics of the diffusor particles. From the results, the $\mathrm{ZnO}$ particles are proven to have positive influences on the development of lighting quality. Additionally, the article analyzed and presented the effects of $\mathrm{ZnO}$ concentration which fluctuates from $2 \%$ to $22 \%$ on the color homogeneity. Thus, the color uniformity is influenced not only by the particle size but also by the concentration of the added $\mathrm{ZnO}$. Hence, managing the color uniformity of WLEDs means controlling the size and concentration of $Z n O$. With the concentration of $10 \% \mathrm{ZnO}$, the lumen output of LEDs reaches the highest values. Meanwhile, when the concentration and size of $\mathrm{ZnO}$ are $14 \%$ and $500 \mathrm{~nm}$ respectively, $\triangle C C T$ is reduced to the lowest value. Based on the manufacturers' requirements, the
\end{abstract}

most appropriate $\mathrm{ZnO}$ concentration and particle size can be determined. However, if requirements include both lumen and color uniformity, the right choice is $14 \%$ concentration with 500 $n m$ particle size of $\mathrm{ZnO}$.

\section{Keywords}

WLEDs, Mie-scattering theory, remote phosphor package, color rendering index, lumen output.

\section{Introduction}

Light-emitting diodes (LEDs) have many benefits for lighting solution because its component materials are strong and stable, cost-saving, and environment-friendly. Thus, it has been widely used in many indoor and outdoor applications as a solid-state light source which can perfectly replace the conventional lights such as light bulbs and discharge lamps [1,2]. So far, white LEDs 
are fabricated from a compound of Indium Gallium Nitride (InGaN) with phosphor materials. This combination is capable of converting blue light to yellow light [3], for which these WLEDs are also known as phosphor-converted white LEDs (pc-WLEDs) [4].

In recent years, many researchers put a lot of efforts into improving the light extraction of WLEDs [5]. They have conducted various analysis about how the packing affect the light performance of pc-WLEDs through comparisons among different LED structures such as conformal coating, half-dome glass cover, isolating configuration, and many more [6]. The main purpose of analyzing is to figure out the impacts of the particle size and particle number of the applied phosphors on the LED output [7]. Beside the perk of being reusable, the particle number is one of the most vital factors influencing lumen output and the color temperature of the lighting device [8]. This state was explained and proved through experiments and model simulation, applying the Monte Carlo method, analyzing the diffusion properties, the absorbing capacity, the converted light, the structural arrangement and the refractive index of materials in the configuration [9]. These approaches were proposed to analyze different phosphors from YAG, Silicate, to green YAG [10]. Moreover, the back-scattering event that causes light loss due to the emitted light being reabsorbed was also studied. The attain results allow researchers to succeed in simulating any type of pc-WLED optical properties.

In the literature of many previous researches, the importance of the color uniformity is not taken into account [11, 12]. There is an obstacle that blocks the way of achieving the high color uniformity for white WLEDs, the yellow ring phenomenon. This phenomenon occurs as the blue light which is strongest surrounding the center joins with phosphor molecule that has consistent strength in all positions [13]. Once this yellow ring appears, it indicates that the high temperature color such as blue light is stronger in the central region and becomes weaker as it reaches the edge and results in light turn more yellow. In addition to that, the angular CCT deviation (DCCTD) can reach up to $3000 \mathrm{~K}$ in lighting configuration that has no adjustment [14], which becomes more drastic when it comes to the use of large size LED applications in illuminating an important surface. Therefore, several studies proposed some solutions to minimize DCCTD, including change the diffusing method to conformal coating [15], applying specialized lens [16], re-modelling the phosphor material. Additionally, there are some complex approaches demonstrated in previous papers to solve this problem such as applying engraved sapphire substrate structure or a graded refractive index with multiple layers of phosphor [17]. However, it is essential to consider the impacts of all the mentioned solutions on DCCTD and lumen output simultaneously. It turns out that the influences are not the same for all the CCT $[18,19]$. Specifically, for high CCT, the DCCTD seems to be stronger than that at low CCT due to the weaker scattered light from phosphor. Regarding that issue, this article proposes a detail analysis of the influence on the DCCTD of $\mathrm{ZnO}$ molecule, an effective scattering particle that is applied in the lighting structure [20]. Though there is no discussion about the phosphor reduction, the rise in backward scattering effect of zirconia seems to be caused by using nano-size particle in remoting configuration [21]. In some cases, the luminous flux could get benefits from this increase, basically an effect of emitted light being recycled. However, the issue is only lumen output is accounted to the influences of $\mathrm{ZnO}$ in their study and correlated color temperature (CCT) deviation was not mentioned [22]. Our simulated pc-WLEDs package is the one constructed with a hemisphere, and this is one of the most popular commercially-used structures. Besides, we will compare experimental and modeling results and analyze them in accordance with the change of the CCT. Moreover, the analysis about not only the chromatic performance but also $\mathrm{ZnO}$ influences based on the concentration of particles at equal color temperature was conducted and demonstrated. The results showed that the phosphor concentration can directly adjust the heating performance of the lighting device. Therefore, it seems that having a decrease in the amount of phosphor may become a crucial part of improving the performance of WLEDs. Generally, phosphor consists of rare earth elements which is considered as critical raw elements. Most of them are cerium, and in some specific phosphors, those elements are europium, 
all of which are considered as critical raw elements. Thus, processes that can reduce the use of rare earth element will be a great topic about this concept.

\section{Research method}

\subsection{Physical and optoelectronic characterization}

A picture of the actual 9-chip WLEDs and the schematic of WLED package with $\mathrm{ZnO}$ base are shown in Fig. 1. A GaN LED chip that emits blue light is applied to the model $453 \mathrm{~nm}$ emitting frequency and peak intensity of $1 \mathrm{~W}$ with running power of $339 \mathrm{~mA}$. The LED chip were cover by a composition of YAG:Ce yellow phosphor (diameter, $13 \pm 2 \mu \mathrm{m}$ ) and silicone. The actual parameters of LED chip are presented in Tab. 1.

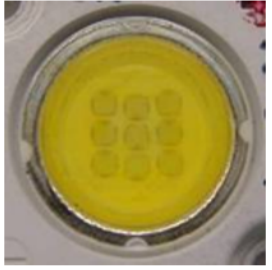

(a)

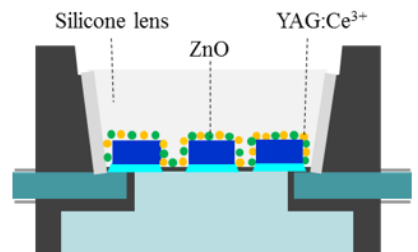

(b)
Fig. 1: (a) Photograph of $9 \mathrm{~W}$ wLED device.

(b) Schematic cross-sectional view of $\mathrm{ZnO}$-doped WLED devices.

\subsection{Mie-scattering analysis}

The Monte Carlo simulation of scattering model can determine the scattering property of quantum-dot-converted elements (QDCEs), similar to the white LEDs that convert phosphor material into energy [23]. There are two vital parameters in this analysis, scattering coefficient and scattering phase function. The scattering coefficient $\mu_{\text {sca }}$ presents the scattering probability, which can express as the following expression [24]:

$$
\mu_{s c a}(\lambda)=\frac{c}{\bar{m}} \int f(D) C_{s c a}(D, \lambda) d D
$$

Tab. 1: The actual parameters of LED chip.

\begin{tabular}{|c|c|}
\hline LED vender & Epistar \\
\hline LED chip & V45H \\
\hline Voltage (V) & $3.5 \sim 3.6$ \\
\hline $\begin{array}{c}\text { Pavelength } \\
\text { (nm) }\end{array}$ & 453 \\
\hline Power (mW) & $320 \sim 340$ \\
\hline Lead frame & 4.7 mm \\
\hline Die attach & Sumitech Size-S \\
\hline & $1295 \mathrm{SA}$ \\
\hline Bonding diagram & \\
\hline
\end{tabular}

In which, $c / \bar{m}$ indicates the particle distribution of the QDs; $c$ is the amount of QD $\left(\mathrm{mg} / \mathrm{cm}^{3}\right)$; $D$ shows the magnitude of the molecule $(\mathrm{nm})$; $\lambda$ represents wavelength $(\mathrm{nm}) ; f(D)$ is the function for QD particle size distribution; $\bar{m}$ is the QD weight $(\mathrm{mg})$ of QDCE, that is a results of $f(D)$ integration. $C_{s c a}(D, \lambda)$ is the scattering cross-section of the QD. Besides, $C_{s c a}(D, \lambda)$ can be written as:

$$
C_{s c a}(D, \lambda)=\frac{P_{s c a}(D, \lambda)}{P_{\text {inc }}(\lambda)}=\frac{\int P_{s c a}(\theta, D, \lambda) d \theta}{P_{i n c}(\lambda)}
$$

In which, $P_{\text {inc }}(\lambda)$ means the incident irradiance of source $\left(\mathrm{W} / \mathrm{m}^{2}\right) ; P_{\text {sca }}(D, \lambda)$ represents the energy emission (W) as light transmits pass the QD; and $P_{s c a}(\theta, D, \lambda)$ is the scattering power (W). While, the other parameter, the scattering phase function describes the scattered power allocation satisfying the standardizing requirements, which can be expressed by the following equation:

$$
\begin{aligned}
& \rho(\theta, \lambda) \\
& =\frac{\int f(D) \rho_{s c a}(\theta, D, \lambda) / P_{s c a}(D, \lambda) C_{s c a}(D, \lambda) d D}{\int f(D) C_{s c a}(D, \lambda) d D}
\end{aligned}
$$




\section{Results and analysis}

The graph in Fig. 2 illustrates the scattering cross-section of the $\mathrm{ZnO}$ particles, $C_{s c a}(D, \lambda)$, with different sizes from $400 \mathrm{~nm}$ to $600 \mathrm{~nm}$. As can be seen, when the size of $\mathrm{ZnO}$ particles increase, $C_{\text {sca }}(D, \lambda)$ also goes up, and then, leading to stronger scattering ability. It seems that with larger particles of $\mathrm{ZnO}$, lights will transmit straight, and this is an advantage for better luminous flux. Meanwhile, when it comes to small particles, there are more light scattering events occurring in all directions, which is beneficial to the color uniformity but disadvantageous to the luminous flux. Moreover, dependent values $C_{\text {sca }}(D, \lambda)$ is in inverse proportion to the wavelength values, which means $C_{s c a}(D, \lambda)$ decrease along with the increase of the wavelength values. From all the charts of Fig. 2, $C_{\text {sca }}(D, \lambda)$ reaches the highest value in the wavelength range of 380 nm.

In Fig. 3, obviously, $\mu_{s c a}(\lambda)$ has the same trend as $C_{\text {sca }}(D, \lambda)$, which is directly proportional to the particles size of $\mathrm{ZnO}$ but inversely proportional to the wavelength values. Specifically, $\mu_{\text {sca }}(\lambda)$ rises with the growth of $\mathrm{ZnO}$ sizes and decreases when the wavelength range is wider. Moreover, the maximum value of $\mu_{\text {sca }}(\lambda)$ is also shown in the $380 \mathrm{~nm}$ wavelength. That the increase of $\mu_{s c a}(\lambda)$ with bigger $\mathrm{ZnO}$ particle proved the better scattering ability of $\mathrm{ZnO}$. The scattering ability of $\mathrm{ZnO}$ can be demonstrated based on $C_{s c a}(D, \lambda)$ and $\mu_{s c a}(\lambda)$, as follows:

1. It is essential to improve the scattering ability in the phosphor layer in order to get the light rays mixed more times and leads to the white light emit color copper.

2. The scattering is maximum at $380 \mathrm{~nm}$ and then gradually decreases. Then, it reaches the smallest value at $780 \mathrm{~nm}$. However, the LED chip has the wavelength of $453 \mathrm{~nm}$; in other words, $\mathrm{ZnO}$ particle benefit the process of increasing the scattering ability of phosphor layers.

Clearly, Fig. 2 and Fig. 3 proved that as $\mathrm{ZnO}$ size is larger, the scattering ability of the phosphor layer becomes better. However, not all

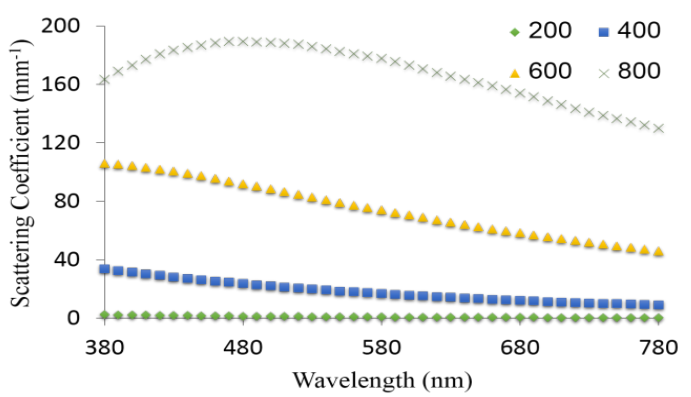

Fig. 2: Scattering coefficient of $\mathrm{ZnO}$ particles with various sizes.

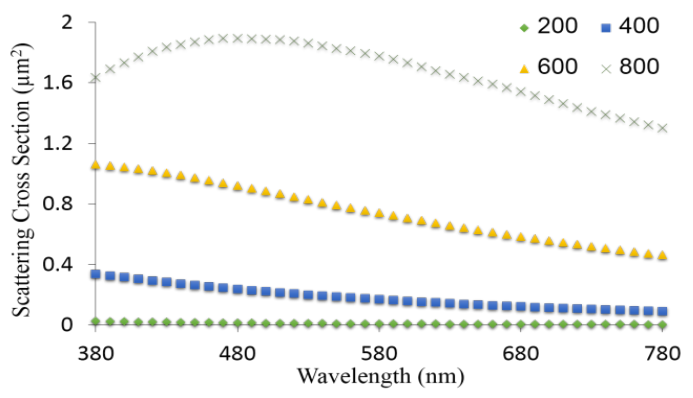

Fig. 3: Scattering cross-sections of $\mathrm{ZnO}$ particles with various sizes.

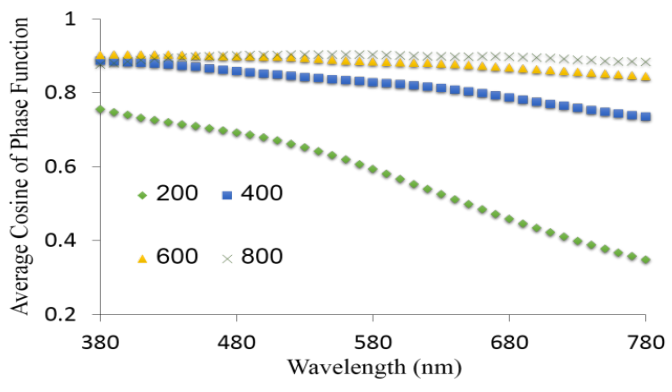

Fig. 4: Average cosine of phase function of $\mathrm{ZnO}$ particles with various sizes.

large size of $\mathrm{ZnO}$ particles are suitable for LED applications with high demand of color uniformity. Thus, $\rho(\theta, \lambda)$, an index describing the angle of scattering intensity, also needs consideration. In Fig. 4 are the values of $\rho(\theta, \lambda)$ in connection with different sizes of $\mathrm{ZnO}$ particles. Obviously, from those charts, the larger the $\mathrm{ZnO}$ particle sizes are, the higher the intensity of scattering becomes. However, with that increase in particle sizes, the scattering angle is narrower. In more detail, $\mathrm{ZnO}$ large-sized particles let the light transmit straight through the particles, and thus the emitted luminous flux 
is benefited. Meanwhile, as the particle size is smaller, the light is distributed in many directions, or in other words, the scattering angle is larger. Moreover, the back-scattering events of light to the LED chip occurring more inside the LEDs package, leading to a reduction in light energy, and as a result, the lumen output is lowered. However, the more the light scattering happens, the more the time that blue and yellow beams are mixed together, resulting in better quality of white light. Specifically, small particle sizes of $\mathrm{ZnO}$ help blue rays scatter more times inside the LEDs, and be distributed to more sides of the LED chip. From this phenomenon, the blue rays combine with "yellow ring" to generate white light, which reduces the "yellow ring" phenomenon, and better the color uniformity.

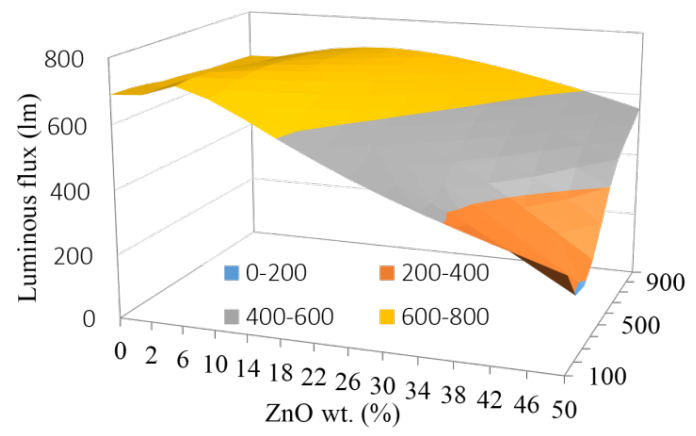

Fig. 5: Lumen output of WLED as a function of the size and concentration of $\mathrm{ZnO}$ particles.

However, if we just based on the results about the sizes of $\mathrm{ZnO}$ particles, it is impossible to give an exact evaluation about the lumen output of the LEDs since it also is affected by the concentration of $\mathrm{ZnO}$ in the phosphor layer. The effects of $\mathrm{ZnO}$ concentrations along with its particle sizes on the lumen output are illustrated in Fig. 5. The lumen output shows a downward trend when the $\mathrm{ZnO}$ concentration has an upward trend, regardless of the $\mathrm{ZnO}$ particle size. On the other hand, the increase of $\mathrm{ZnO}$ concentration is beneficial to the scattering capacity, from which the energy of emitted light can be reduced. Thus, the right selections of size and concentration of $\mathrm{ZnO}$ particles play a crucial role in WLED fabrication. From the results of Fig. 5 , it is possible to choose the concentration of $\mathrm{ZnO}$ at around $10 \%$, regardless of the particle sizes, for accomplishing the greatest lumen output. Nevertheless, the study focuses on not only the lumen output, but also the color uniformity of the WLEDs. Therefore, an analysis of the results from Fig. 6 can help to figure out the most suitable concentration and particle size of $\mathrm{ZnO}$ to achieve both targets.

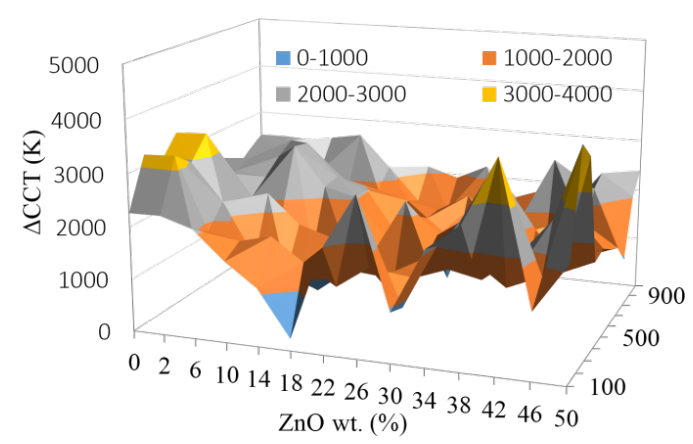

Fig. 6: $\triangle \mathrm{CCT}$ of WLED as a function of the size and concentration of $\mathrm{ZnO}$ particles.

As can be seen, when $\mathrm{ZnO}$ concentration is about $14 \%, \Delta \mathrm{CCT}$ declines considerably though its value is not the lowest one. However, with the $500 \mathrm{~nm}$ particle size of $\mathrm{ZnO}$ and at $14 \%$ $\mathrm{ZnO}$ concentration, $\triangle \mathrm{CCT}$ reaches the lowest point. Nevertheless, when the concentration of $\mathrm{ZnO}$ continues to grow from the point of $14 \%$, it seems that $\triangle \mathrm{CCT}$ also shows its increase. This can be understood by the strong scattering events in the phosphor layer. As a result, there are more and more scattered blue light, especially with smaller size of $\mathrm{ZnO}$ particles. However, to achieve a high color uniformity, the balance between the intensity of blue and yellow lights is very important, which means that the level of $\triangle \mathrm{CCT}$ must be lowered. Thus, the $\mathrm{ZnO}$ particle size and concentration must be adjusted. If the yellow light emitted is more than the blue one, the "yellow ring" phenomenon will occur, leading to generating warm white light. Therefore, it needs to get the scattering of blue light improved in order to reach the reduction of that phenomenon and achieve a balance in the amount of blue and yellow light.

By investigating the scattering ability of the phosphor layer through values $C_{s c a}(D, \lambda)$, 
$\mu_{\text {sca }}(\lambda)$ and $\rho(\theta, \lambda)$ with the addition of $\mathrm{ZnO}$, the suitable $\mathrm{ZnO}$ particle parameters could be determined, depending on what the manufacturers require. If the target is to get high a lumen output, it is possible to add $14 \% \mathrm{ZnO}$ into the phosphor layer. Meanwhile, the manufacturers want to accomplish a WLED package with high color uniformity, $14 \%$ turns out to be the most suitable concentration of $\mathrm{ZnO}$ for application. In case of that both luminous flux and color homogeneity are the goal, $14 \%$ concentration can be selected with $500 \mathrm{~nm} \mathrm{ZnO}$ particle sizes.

\section{Conclusions}

In short, the influences of $\mathrm{ZnO}$ particles on the lumen output and color homogeneity of WLED are performed and demonstrated in this article. For carrying out experiments and simulations, $\mathrm{ZnO}$ particles whose size is from $400 \mathrm{~nm}$ to $600 \mathrm{~nm}$ are added into YAG:Ce phosphor layer. The purpose of mixing $\mathrm{ZnO}$ and YAG:Ce is to enhance the scattering ability of the phosphor layer, resulting in a better color uniformity. In addition, the aim of the studies is to analyze and propose the most suitable $\mathrm{ZnO}$ size and concentration for WLED applications. Based on the analysis of $\mathrm{C}_{s c a}(D, \lambda), \mu_{s c a}(\lambda)$ and $\rho(\theta, \lambda)$, the study gives a clear detailed demonstration about the scattering effect of $\mathrm{ZnO}$ particles by size, in which the larger the size of $\mathrm{ZnO}$ particles is, the greater the scattering ability becomes. Besides, the impacts of $\mathrm{ZnO}$ concentration are also included in the paper, so manufactures can make their own choice about how they apply $\mathrm{ZnO}$ to their production process. The results showed that when $\mathrm{ZnO}$ concentration is $10 \%$, the lumen output of LEDs has the highest value. Meanwhile, as $\mathrm{ZnO}$ concentration and particle size of $14 \%$ and $500 \mathrm{~nm}$, respectively, $\Delta \mathrm{CCT}$ value is the lowest. However, based on the requirements of the production, manufacturers can decide the most appropriate parameters of $\mathrm{ZnO}$ for application. If they want to better both lumen output and color uniformity, it is possible to add $14 \%$ and $500 \mathrm{~nm} \mathrm{ZnO}$ into the phosphor layers of WLED bulks.

\section{Acknowledgments}

This work was supported by the Ministry of Science and Technology of the Republic of China, project 108-2622-E-992-013-CC3.

\section{References}

[1] Nguyen, D. Q. A., Le Phan, X., \& Lee, H. Y. (2019). Enhanced Luminous Flux of White Led using Flat Dual-layer Remote Phosphor Configuration. Journal of Advanced Engineering and Computation, $3(2), 425-431$.

[2] Yu, Y., Cao, C., Wu, Z., Wu, Q., Lin, W., Peng, X., ... \& Tong, Q. (2019). Improving the color-rendering index of a tandem warm white organic light-emitting device by employing a simple fabrication process. Optics Letters, 44(4), 931-934.

[3] Yeh, C. T., Chou, Y. I., Yang, K. S., Wu, S. K., \& Wang, C. C. (2019). Luminescence material characterizations on laserphosphor lighting techniques. Optics express, 27(5), 7226-7236.

[4] Chang, Y. P., Chang, J. K., Chen, H. A., Chang, S. H., Liu, C. N., Han, P., \& Cheng, W. H. (2019). An advanced laser headlight module employing highly reliable glass phosphor. Optics express, 27(3), 1808-1815.

[5] Sharma, S., Brahme, N., Bisen, D. P., \& Dewangan, P. (2018). Cool white light emission from $\mathrm{Dy}^{3+}$ activated alkaline alumino silicate phosphors. Optics express, 26(22), 29495-29508.

[6] Lee, H., Cho, H., Byun, C. W., Han, J. H., Kwon, B. H., Choi, S., ... \& Cho, N. S. (2018). Color-tunable organic lightemitting diodes with vertically stacked blue, green, and red colors for lighting and display applications. Optics express, 26(14), 18351-18361.

[7] Kim, W. J., Kim, T. K., Kim, S. H., Yoon, S. B., Jeong, H. H., Song, J. O., 
\& Seong, T. Y. (2018). Improved angular color uniformity and hydrothermal reliability of phosphor-converted white lightemitting diodes by using phosphor sedimentation. Optics express, 26(22), 28634-28640.

[8] Li, B., Annadurai, G., Sun, L., Liang, J., Wang, S., Sun, Q., \& Huang, X. (2018). High-efficiency cubic-phased blue-emitting $\mathrm{Ba}_{3} \mathrm{Lu}_{2} \mathrm{~B}_{6} \mathrm{O}_{15}: \mathrm{Ce}^{3+}$ phosphors for ultraviolet-excited white-lightemitting diodes. Optics Letters, 43(20), 5138-5141.

[9] Peng, Y., Guo, X., Li, R., Cheng, H., \& Chen, M. (2017). Thermally stable WLEDs with excellent luminous properties by screen-printing a patterned phosphor glass layer on a microstructured glass plate. Applied Optics, 56(12), 3270-3276.

[10] Zhang, W., Yang, W., Zhong, P., Mei, S., Zhang, G., Chen, G., ... \& Guo, R. (2017). Spectral optimization of color temperature tunable white LEDs based on perovskite quantum dots for ultrahigh color rendition. Optical Materials Express, 7(9), 3065-3076.

[11] Sahu, I. P., Bisen, D. P., \& Tamrakar, R. K. (2016). Dysprosium-Doped Strontium Magnesium Silicate White Light Emitting Phosphor Prepared by Solid State Reaction Method. Journal of Display Technology, 12(11), 1478-1487.

[12] Singh, V. K., Tripathi, S., Mishra, M. K., Tiwari, R., Dubey, V., \& Tiwari, N. (2016). Optical Studies of Erbium and Ytterbium Doped $\mathrm{Gd}_{2} \mathrm{Zr}_{2} \mathrm{O}_{7}$ Phosphor for Display and Optical Communication Applications. Journal of Display Technology, 12(10), 1224-1228.

[13] Anxiang, G., Fuwang, M., Peican, C., Yue, G., Qian, C., Liya Z. (2016). Photoluminescence Properties and Energy Transfer of $\mathrm{Eu}^{3+}, \mathrm{Bi}^{3+} \mathrm{Co}-$ Doped $\mathrm{Ca}_{9} \mathrm{Y}\left(\mathrm{PO}_{4}\right)_{7}$ Phosphors. Journal of Display Technology, 12(2), 136-142.

[14] Hu, C., Shi, Y., Feng, X., \& Pan, Y. (2015). YAG:Ce/(Gd,Y)AG:Ce duallayered composite structure ceramic phos- phors designed for bright white lightemitting diodes with various CCT. Optics Express, 23(14), 18243-18255.

[15] Chung, H. C., Te H. L., Han Y. L., Hung Y. K., Sheng Y. C. (2015). Effects of Flux Additives on Characteristics of $\mathrm{Y}_{2.95} \mathrm{Al}_{5} \mathrm{O}_{12}$ : $0.05 \mathrm{Ce}^{3+}$ Phosphor: Thermal Stability and Application to WLEDs. Journal of Display Technology, 11(5), 466-470.

[16] Tang, Y. R., Zhou, S. M., Yi, X. Z., Lin, H., \& Zhang, S. (2015). Microstructure optimization of the composite phase ceramic phosphor for white LEDs with excellent luminous efficacy. Optics letters, 40(23), 5479-5481.

[17] Rao, L., Tang, Y., Li, Z., Ding, X., Li, J., Yu, S., ... \& Lu, H. (2017). Effect of $\mathrm{ZnO}$ nanostructures on the optical properties of white light-emitting diodes. Optics express, 25(8), A432-A443.

[18] Li, J., Li, Z., Liang, G., Yu, S., Tang, Y., \& Ding, X. (2016). Color uniformity enhancement for COB WLEDs using a remote phosphor film with two freeform surfaces. Optics Express, 24(21), 23685-23696.

[19] Jeong, H., Salas-Montiel, R., \& Jeong, M. S. (2015). Optimal length of $\mathrm{ZnO}$ nanorods for improving the light-extraction efficiency of blue InGaN light-emitting diodes. Optics express, 23(18), 23195-23207.

[20] Jeon, J. H., Choi, P. J., Oh, S. J., Kang, Y. J., Kim, J. Y., \& Kwon, M. K. (2015). Improvement of the light extraction efficiency of InGaN/GaN blue light emitting diodes using $\mathrm{ZnO}$ nanostructures. Journal of Nanoscience and Nanotechnology, 15(7), 5215-5219.

[21] Tang, Y., Li, Z., Li, Z. T., Li, J. S., Yu, S. D., \& Rao, L. S. (2017). Enhancement of luminous efficiency and uniformity of CCT for quantum dot-converted LEDs by incorporating with $\mathrm{ZnO}$ nanoparticles. IEEE Transactions on Electron Devices, 65(1), 158-164. 
[22] Liu, L., Tan, X., Teng, D., Wu, M., \& Wang, G. (2015). Simultaneously Enhancing the Angular-Color Uniformity, Luminous Efficiency, and Reliability of White Light-Emitting Diodes by $\mathrm{ZnO} \mathrm{SiO}_{2}$ Modified Silicones. IEEE Transactions on Components, Packaging and Manufacturing Technology, 5(5), 599-605.

[23] Kim, S. H., Song, Y. H., Jeon, S. R., Jeong, T., Kim, J. Y., Ha, J. S., ... \& Park, H. J. (2013). Enhanced luminous efficacy in phosphor-converted white vertical lightemitting diodes using low index layer. Optics Express, 21(5), 6353-6359.

[24] Chen, L. Y., Chang, J. K., Wu, Y. R., Cheng, W. C., Chen, J. H., Tsai, C. C., \& Cheng, W. H. (2013). Optical model for novel glass-based phosphor-converted white light-emitting diodes. Journal of Display Technology, 9(6), 441-446.

\section{About Authors}

Ming Jui CHEN was born in Tainan city, Taiwan. He has been working at the Department of Electrical Engineering, National Kaohsiung University of Science and Technology, Kaohsiung, Taiwan. His research interest is optical material.
Van Tho LE was born in Thanh Hoa province, Vietnam. In 2019, he received his master degree from the University of Science - Vietnam National University. His research interest is optoelectronics. He has worked at Institute of Tropical Biology, Vietnam Academy of Science and Technology.

Doan Quoc Anh NGUYEN was born in Khanh Hoa province, Vietnam. He has been working at the Faculty of Electrical and Electronics Engineering, Ton Duc Thang University. Quoc Anh received his PhD degree from National Kaohsiung University of Science and Technology, Taiwan in 2014. His research interest is optoelectronics.

Thinh Cong TRAN has been working at the Faculty of Electrical and Electronics Engineering, Ton Duc Thang University. Dr Thinh received his $\mathrm{PhD}$ degree from Technical University of Ostrava, Czechia in 2018. His research interest is optoelectronics. 